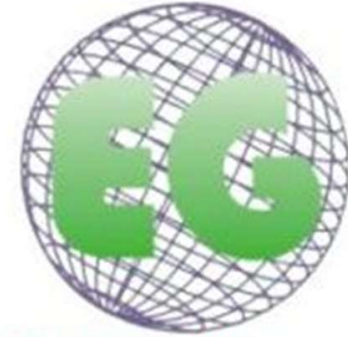

ISSN 1695-6141 N58

\title{
Revisión sistemática: influencia de los Grupos de Ayuda Mutua sobre cuidadores familiares
}

Systematic review: Influence of Self-Help Groups on family caregivers

\author{
Claudia Bernabéu-Álvarez ${ }^{1}$ \\ Mar Faus-Sanoguera ${ }^{2}$ \\ Marta Lima-Serrano ${ }^{3}$ \\ Joaquín-Salvador Lima-Rodríguez ${ }^{3}$
}

1 Enfermera Especialista en Enfermería Familiar y Comunitaria. Doctoranda por la Universidad de Sevilla. Facultad de Enfermería, Fisioterapia y Podología. Sevilla. España. claberalv@gmail.com

2 Enfermera. Hospital General Mateo Orfila. Menorca. España.

${ }^{3}$ Enfermera. PhD Profesor Asociado. Facultad de Enfermería, Fisioterapia y Podología. Universidad de Sevilla. Sevilla. España.

\section{https://doi.org/10.6018/eglobal.392181}

Recibido: $13 / 08 / 2019$

Aceptado: 16/01/2020

\section{RESUMEN:}

Introducción: Desde la incorporación de la mujer al mundo laboral, junto con el envejecimiento progresivo de la población y el aumento de las enfermedades crónicas, se produce una alteración en el rol del cuidador, debido a la carga física, laboral y familiar que soporta, generándose conflictos emocionales y con el resto de miembros de la familia. La ayuda mutua puede ser una alternativa eficaz para promover el bienestar de los cuidadores, así como el de sus familias y dependientes.

Objetivo: Conocer las características de los grupos de ayuda mutua (GAM) para cuidadores familiares y su influencia sobre los cuidadores, la persona dependiente y la salud familiar.

Metodología: Revisión sistemática. La estrategia de búsqueda incluyó las bases de datos Pubmed, Scopus, Psycinfo, Eric, Cochrane plus y CSIC. Se buscaron artículos escritos en español, catalán, inglés, portugués o francés, publicados en los últimos 10 años.

Resultados: Se seleccionaron 12 artículos relacionados con el tema de estudio. Todos los estudios muestran que la participación en estos grupos puede mejorar el bienestar físico-psicológico, el estado de salud de los cuidadores y, al mismo tiempo, reforzar su sentimiento de apoyo social, aunque faltan estudios en nuestro medio, con un tamaño muestral superior y de mayor calidad.

Conclusiones: Los cuidadores se benefician de participar en grupos de ayuda mutua. Por lo tanto, deben convertirse en un componente de rutina del cuidador familiar.

Palabras clave: Cuidador familiar, Grupos de ayuda mutua (GAM), salud familiar, sobrecarga del cuidador, personas dependientes.

\section{ABSTRACT:}

Introduction: Since the incorporation of women into the world of work, together with the progressive ageing of the population and the increase of chronic diseases, there is an alteration in the role of the caregiver, due to the physical, work and family burden it bears, emotional conflicts and with other family members. Mutual aid can be an effective alternative to promoting the well-being of caregivers, as well 
as their families and dependents.

Objective: To know the characteristics of self-help groups for family caregivers and their influence on caregivers, the dependent and family health.

Methodology: Systematic review. The search strategy included the Pubmed, Scopus, Psycinfo, Eric, Cochrane plus and CSIC databases; selecting scientific articles in either Spanish, Catalan, English, Portuguese or French, for the last 10 years.

Results: 12 articles related to the study topic were selected. All studies show that participation in these groups can improve the physical-psychological well-being, the health of caregivers and, at the same time, reinforce their sense of social support, although there is a lack of studies in our environment, with a size higher quality sample.

Conclusions: Caregivers benefit from participating in self-help groups. Therefore, they should become a routine component of the family caregiver.

Keywords: Family caregiver, self-help groups, family health, caregiver burden, dependents.

\section{INTRODUCCIÓN}

La enfermedad o dependencia de un miembro de la unidad familiar puede llegar a formar parte de la vida familiar, produciendo cambios estructurales, procesuales y emocionales. El $88 \%$ del tiempo total dedicado al cuidado de la salud se provee en el ámbito doméstico, formando parte de la vida cotidiana de las familias. El cuidado familiar lo asume generalmente una sola persona, casi siempre una mujer ${ }^{(1)}$. El cuidador es la persona encargada del cuidado de estos enfermos, dedicando en su mayoría las $24 \mathrm{~h}$ a él. Poco a poco va descuidando sus propias necesidades para ocuparse por completo de éste. El cuidado de un familiar dependiente puede afectar negativamente en el ámbito social, familiar del cuidador, y la vida profesional (2). Además existe un peligro en su salud física, un posible aumento en el aislamiento social y un aumento del riesgo de ansiedad y de los trastornos depresivos, causando así sentimientos de culpa en el proceso de cuidado, por lo que puede llegar a poner en peligro en última instancia su capacidad de funcionar como cuidador, por lo que también el estrés del cuidador está vinculado a los resultados negativos en el receptor de la atención y a los costes para la sociedad, como el aumento de hogares de ancianos y los ingresos hospitalarios ${ }^{(3)}$. Además, puede afectar a la salud familiar entendida ésta como su capacidad de funcionar y adaptarse a acontecimientos vitales estresantes, como pueden ser la enfermedad o discapacidad de alguno de sus miembros ${ }^{(4)}$.

En Andalucía se estima que hay alrededor de 130.000 personas cuidadoras de enfermos y personas mayores. (5) Tras la aprobación del Decreto de Apoyo a las Familias Andaluzas, las personas cuidadoras han pasado a ser una pieza imprescindible en nuestro Sistema Sanitario realizando una gran labor en el ámbito social y familiar con la atención que prestan a familiares discapacitados. Por ello, se ha puesto en marcha un programa específico para proporcionar ayuda a las cuidadoras familiares, el "Plan de Mejora de la atención a personas cuidadoras en Andalucía", con intervenciones como el apoyo formal, mediante servicios comunitarios «de respiro»; programas psicoeducativos; grupos de ayuda mutua; intervenciones psicoterapéuticas (o counseling); o una combinación de algunas de las anteriores. ${ }^{(6)}$

Uno de estos recursos con los que pueden contar las familias, en estas situaciones, son los grupos de ayuda mutua (GAM), en los que se intercambian, en un ambiente terapéutico, estrategias de afrontamiento y se buscan soluciones apropiadas. (7) Un grupo de ayuda mutua es definido como un grupo de "iguales" para ayudarse unos a otros, ofrecer apoyo emocional, facilitar la adquisición de habilidades y el manejo de 
herramientas para enfrentarse y solucionar los problemas que tienen en relación a los cuidados, aumentar su autoestima y disminuir la codependencia, ayudar en el proceso de adaptación social necesario en la situación de cuidados teniendo al grupo como red de apoyo social, disminuir el consumo farmacológico por sintomatología relacionada con la sobrecarga de la persona cuidadora. ${ }^{(8)}$

Respecto a las modalidades de grupos de familiares, se diferenciaron tres clases de grupos: informativos, de autoayuda y de apoyo. Los grupos informativos tienen como principal objetivo ayudar a las familias a cuidar mejor al enfermo a través de información médica y/o de atención de enfermería. Su duración es corta (6-10 sesiones). Los GAM se fundamentan en el apoyo entre sus miembros, si bien, éstos han ido evolucionando a ser guiados por un profesional y definiéndose como grupos de apoyo, donde se aúnan los aspectos anteriores de información y de apoyoorientación y aprendizaje de estrategias. ${ }^{(9)}$

El impacto cada vez mayor que el cuidado de las personas mayores dependientes tiene sobre la sociedad se ve reflejado en el número de publicaciones que se vienen realizando sobre intervenciones para cuidadores. Si bien el diseño y los procedimientos de estas intervenciones han ido ganando en calidad, la realidad es que, en el momento actual, el tamaño del efecto de las intervenciones para reducir el malestar de los cuidadores es, en el mejor de los casos, moderado. Los estudios realizados en los últimos veinte años permiten afirmar hoy que los cuidadores informales se ven expuestos a una situación de estrés que incrementa el riesgo de padecer diversos problemas físicos y emocionales, especialmente ansiedad y depresión. Hasta la fecha se han desarrollado distintos tipos de intervenciones. Sin embargo, no es abundante ni la aplicación de programas de apoyo, ni la investigación sobre la eficacia de estos programas, especialmente en nuestro contexto sociocultural. ${ }^{(10)}$

El estudio empírico de intervenciones dirigidas a apoyar a los cuidadores de mayores dependientes comenzó en los años 80 , sin que todavía se haya conseguido establecer con claridad sus ventajas. (5) En un intento de establecer la eficacia de estas intervenciones en la mejora del estado emocional de los cuidadores, se han llevado a cabo varias revisiones. Sin embargo, todas ellas se centran única y exclusivamente en cuidadores de personas con demencia, ${ }^{(8)}$ aunque en 2017, un metaanálisis recogió estudios muy variados, sobre personas dependientes por distintas causas (enfermedades crónicas, enfermedades dependientes, personas mayores...) o artículos centrados, en general, en los cuidadores familiares, pero mediados todos por GAM online. (11)

Por lo tanto, esta búsqueda de la literatura sobre los GAM para cuidadores familiares trata de conocer las características de estos GAM y su influencia sobre los cuidadores, la persona dependiente y la salud familiar.

\section{METODOLOGÍA}

Se ha llevado a cabo una revisión sistemática de la literatura. Para realizar este proceso, se han seguido las recomendaciones de la declaración PRISMA ${ }^{(12)}$.

Se seleccionaron las bases de datos Pubmed(13), SCOPUS(14), Cochrane Plus ${ }^{(15)}$, ERIC (16), Psyclnfo(17), Biblioteca Nacional de Ciencias de la Salud (BNCS) del Instituto 
de Salud Carlos III. (18)

\section{Estrategia de búsqueda}

En primer lugar, para la búsqueda se han usado palabras claves relacionadas con los objetivos deseados, según los términos Mesh y Decs: (Caregiver) OR (home nursing) AND (self-help group) OR (group structure) AND (family health). La estrategia de búsqueda empleada para cada una de las bases de datos con sus respectivos limitadores y registros obtenidos aparecen en la tabla 1.

Tabla 1: Estrategia de búsqueda detallada.

\begin{tabular}{|c|c|c|c|}
\hline Base de datos & $\begin{array}{l}\text { Estrategia de búsqueda } \\
\text { general }\end{array}$ & $\begin{array}{l}\text { Registros } \\
\text { obtenidos }\end{array}$ & $\begin{array}{l}\text { Fecha de } \\
\text { búsqueda }\end{array}$ \\
\hline PUBMED & \multirow{3}{*}{$\begin{array}{c}\text { (caregiver OR home nursing) } \\
\text { AND } \\
\text { (self-help group OR Group Structure) }\end{array}$} & 85 & $04 / 03 / 2019$ \\
\hline SCOPUS & & 89 & $08 / 03 / 2019$ \\
\hline PsycINFO & & 61 & $15 / 03 / 2019$ \\
\hline ERIC & AND & 23 & $08 / 03 / 2019$ \\
\hline Cochrane Plus & \multirow{2}{*}{$\begin{array}{l}\text { LÍMITES: } \\
\text {-Resumen } \\
\text {-Últimos } 10 \text { años } \\
\text {-Idioma* }\end{array}$} & 165 & $08 / 03 / 2019$ \\
\hline $\begin{array}{c}\text { Biblioteca } \\
\text { Nacional de } \\
\text { Ciencias de la } \\
\text { Salud }\end{array}$ & & 1 & $11 / 03 / 2019$ \\
\hline
\end{tabular}

\section{Proceso de selección}

Para la selección de los artículos se tuvieron en cuenta los siguientes criterios de inclusión y de exclusión:

\section{$>$ Criterios de inclusión:}

- Artículo original de investigación.

- Estudios de carácter cuantitativo o mixto.

- Calidad del artículo moderada o fuerte.

- Relación directa con la salud familiar y la salud individual en cuidadores de personas dependientes.

- Idioma: español, catalán, inglés, portugués, o francés.

- Fecha de publicación en los últimos diez años.

> Criterios de exclusión:

- Imposibilidad para recuperar el texto completo del artículo.

- Artículo repetido de una búsqueda anterior.

- Los receptores del cuidado no son personas dependientes.

Para la selección de los artículos se han realizado distintas estrategias de filtrado del total de los artículos encontrados tras la búsqueda, y ésta se ha dividido en dos fases. 
En el primer cribado se han descartado los artículos según el título y el resumen de cada uno de ellos. De los 424 registros encontrados, se seleccionaron inicialmente 69 artículos.

Respecto a los criterios de rechazo en esta primera fase de selección, según el título y resumen, se pueden dividir en:

Idioma alemán o chino.

No relación directa del título con el cuidador, GAM o la salud familiar.

No incluye resumen.

Artículo repetido de una búsqueda anterior.

En la segunda fase, el cribado se realizó a través de la lectura del texto completo, obteniéndose finalmente 12 artículos seleccionados (de los 69 de la fase anterior).

Los motivos de exclusión en esta segunda fase fueron los siguientes:

No es un artículo original de investigación.

La metodología del artículo no es la buscada.

No guarda relación directa con la salud familiar, GAM y el cuidador.

Imposibilidad para recuperar el texto completo del artículo.

Calidad del artículo débil.

Los receptores del cuidado no son personas dependientes.

El proceso de selección completo se ve resumido en el siguiente flujograma.

Figura 1: Resumen del proceso de selección de artículos.

$\mathrm{N}^{\circ}$ de registros obtenidos en cada base de datos (424 artículos)

Pubmed: 85

Scopus: 89

PsycINFO: 61

ERIC: 23

Cochrane plus: 165

BNCS: 1

Fase 1: Cribado según el título y el resumen del artículo (69 artículos)

Fase 2: Cribado según el texto completo y la calidad del artículo (12

Pubmed: 28 artículos)

$\quad$ Scopus: 27
PsycINFO: 1
ERIC: 7
Cochrane plus: 5
BNCS: 1

Pubmed: 5

Scopus: 5

PsycINFO: 0

ERIC: 0

Cochrane plus: 1

BNCS: 1

Las variables de interés utilizadas en la extracción de datos en estos artículos fueron las características de los GAM, las ventajas de los GAM en cuidadores, el impacto de los GAM en la salud familiar y los beneficios de los GAM para la persona dependiente. También se tuvo en cuenta el país, para saber si se desarrolla en una sociedad occidental (individualista) y oriental (grupal).

Una vez seleccionados los artículos que finalmente se incluyeron en la revisión, fueron analizados utilizando el método CASPe (Critical Appraisal Skills Programme; 
en español: Programa de Habilidades en Lectura Crítica). Se trata de una herramienta estructurada en: validez, resultados y aplicabilidad. Cuanto mayor es la puntuación, mayor es la calidad del estudio. (19) Por ello, se tomó como límite sólo dos fallos en el total de ítems sobre calidad del artículo.

\section{RESULTADOS}

La calidad de los artículos, queda detallada en la siguiente tabla (tabla 2).

Tabla 2: Análisis de la calidad de los artículos.

\begin{tabular}{|c|c|c|}
\hline Artículo & Calidad & Descripción detallada del análisis \\
\hline $1^{(20)}$ & $\begin{array}{l}\text { Fuerte } \\
(9 / 10)\end{array}$ & $\begin{array}{l}\text { El punto débil (número 8): al realizarse los distintos estudios } \\
\text { en poblaciones similares entre sí, no se sabe si pueden } \\
\text { aplicarse los resultados en nuestro medio. }\end{array}$ \\
\hline $2^{(21)}$ & $\begin{array}{l}\text { Fuerte } \\
(10 / 11)\end{array}$ & $\begin{array}{l}\text { El punto débil es el número } 9 \text {, ya que no sabemos si pueden } \\
\text { aplicarse los resultados en nuestro medio, debido a que el } \\
\text { estudio se desarrolla en China. }\end{array}$ \\
\hline $3^{(22)}$ & Alta & $27 / 27$ entre bien y muy bien. \\
\hline $44^{(23)}$ & $\begin{array}{l}\text { Fuerte } \\
(10 / 11)\end{array}$ & $\begin{array}{l}\text { Aunque no se mantuvieron ciegos los clínicos, pacientes y } \\
\text { personal de estudio o, al menos, no se refleja. }\end{array}$ \\
\hline $5^{(24)}$ & $\begin{array}{l}\text { Fuerte } \\
(10 / 11)\end{array}$ & $\begin{array}{l}\text { El punto débil: no se mantuvieron ciegos los clínicos y } \\
\text { personal de estudio o, al menos, no lo expresa. }\end{array}$ \\
\hline $6^{(25)}$ & $\begin{array}{c}\text { Alta } \\
(9 / 11- \\
9 / 10)\end{array}$ & $\begin{array}{l}\text { En cuanto a la cuantitativa ( } 9 / 11) \text {. En cuanto a la cualitativa } \\
(9 / 10) \text {, no queda explícito el método elegido (fenomenología, } \\
\text { teoría fundamentada, etnología...). }\end{array}$ \\
\hline $7^{(26)}$ & $\begin{array}{l}\text { Fuerte } \\
(10 / 11)\end{array}$ & $\begin{array}{l}\text { La limitación en este caso es que no se mantuvieron ciegos } \\
\text { los clínicos, pacientes y personal de estudio o, al menos, no } \\
\text { se refleja. }\end{array}$ \\
\hline $8^{(27)}$ & $\begin{array}{l}\text { Buena } \\
(8 / 10)\end{array}$ & $\begin{array}{l}\text { Metodología cualitativa, calidad fuerte }(8 / 10) \text {. Por otro lado, la } \\
\text { calidad de la parte cuantitativa (como estudio observacional } \\
\text { descriptivo transversal), es buena. }\end{array}$ \\
\hline $9^{(28)}$ & $\begin{array}{l}\text { Alta } \\
(9 / 10)\end{array}$ & $\begin{array}{l}\text { Pero sin quedar explícito el método elegido (fenomenología, } \\
\text { teoría fundamentada, etnología...). }\end{array}$ \\
\hline $10^{(29)}$ & $\begin{array}{l}\text { Fuerte } \\
(10 / 11)\end{array}$ & $\begin{array}{l}\text { Pero al tratarse de población china, no puede extrapolarse } \\
\text { por las diferencias socioculturales. }\end{array}$ \\
\hline $11^{(30)}$ & $\begin{array}{l}\text { Fuerte } \\
(10 / 11)\end{array}$ & $\begin{array}{l}\text { Aunque al tratarse de población china, nos encontramos con } \\
\text { el mismo problema que en el artículo anterior. }\end{array}$ \\
\hline $12^{(11)}$ & $\begin{array}{l}\text { Fuerte } \\
(10 / 10)\end{array}$ & $\begin{array}{l}\text { Cumple claramente con todos los apartados, incluso } \\
\text { indicando la falta de estudios de alta calidad. }\end{array}$ \\
\hline
\end{tabular}

Según la clasificación realizada con el método CASPe, los artículos seleccionados tienen un nivel de calidad alto, pero son heterogéneos, ya que los GAM han sido estudiados y utilizados en una gran variedad de países entre todo el mundo, en diferentes años y bases de datos y con diferente metodología.

Por ello, para poder desarrollar los principales resultados estos se agruparán en base a las siguientes categorías: 
1. Las características de los GAM.

2. Ventajas de los GAM en cuidadores.

3. Impacto de los GAM en la salud familiar.

4. Beneficios de los GAM para la persona dependiente.

En la tabla número 3 se muestran los principales autores, el año de publicación y el nivel de evidencia, el tipo de estudio y la muestra, el objetivo a alcanzar de cada uno junto con las categorías mencionadas y el análisis llevado a cabo, y los resultados obtenidos para el posterior análisis.

Para determinar el nivel de evidencia de los artículos se utilizó el esquema de gradación de la Agència d'Avaluació de Tecnologia Mèdica (AATM) de la Generalitat de Catalunya.

Tabla 3: Artículos seleccionados para revisión con sus resultados principales.

\begin{tabular}{|c|c|c|c|}
\hline $\begin{array}{c}\text { Autor. } \\
\text { Año. } \\
\text { Evidencia }\end{array}$ & $\begin{array}{c}\text { Tipo de estudio. } \\
\text { Población. } \\
\text { País }\end{array}$ & $\begin{array}{c}\text { Objetivo. } \\
\text { Categoría. } \\
\text { Análisis }\end{array}$ & $\begin{array}{l}\text { Principales } \\
\text { resultados }\end{array}$ \\
\hline $\begin{array}{l}\text { Candy, } \\
\text { Jones, } \\
\text { Drake, } \\
\text { Leurent, } \\
\text { King } \\
(2011) \\
\text { I }\end{array}$ & $\begin{array}{c}\text { Revisión } \\
\text { sistemática. } \\
11 \text { ensayos } \\
\text { clínicos con } 1836 \\
\text { cuidadores de un } \\
\text { amigo o familiar } \\
\text { con una } \\
\text { enfermedad en } \\
\text { fase terminal. } \\
\text { Reino Unido }\end{array}$ & $\begin{array}{l}\text { Evaluar los efectos de } \\
\text { los GAM en la salud } \\
\text { psicológica y física de } \\
\text { los cuidadores de } \\
\text { pacientes en la fase } \\
\text { terminal de su } \\
\text { enfermedad. } \\
2 \\
\text { Diferencia de medias } \\
\text { estandarizada [DME]; } \\
\text { intervalo de confianza } \\
\text { [IC] del 95\%. }\end{array}$ & $\begin{array}{l}\text { Los GAM pueden } \\
\text { ayudarles a afrontar su } \\
\text { rol de cuidador y } \\
\text { mejorar su calidad de } \\
\text { vida. } \\
\\
7 \text { ensayos: DME }-0,05 ; \\
\text { IC del } 95 \% \text { : }-0,24 \text { a } \\
0,14 ; 6 \text { ensayos: DME } \\
0,08 \text {; IC del } 95 \%:-0,11 \\
\text { a } 0,26\end{array}$ \\
\hline $\begin{array}{c}\text { Chien, } \\
\text { Chan, } \\
(2013) \\
\text { II }\end{array}$ & $\begin{array}{l}\text { Ensayo clínico } \\
\text { controlado } \\
\text { aleatorizado. } \\
135 \text { cuidadores } \\
\text { de familiares con } \\
\text { esquizofrenia. } \\
\text { Dos tercios eran } \\
\text { hombres, y padre } \\
\text { o cónyuge. } \\
\text { China }\end{array}$ & $\begin{array}{c}\text { Evaluar los efectos de } \\
\text { los GAM dirigidos por } \\
\text { familiares de personas } \\
\text { con esquizofrenia en el } \\
\text { funcionamiento familiar y } \\
\text { soporte social. } \\
3,4 \\
\text { Análisis de la varianza } \\
\text { (ANOVA). }\end{array}$ & $\begin{array}{c}\text { Mejoría } \\
\text { significativamente } \\
\text { mayor en el } \\
\text { funcionamiento de la } \\
\text { familia [ } F=5.40, p=.005] \\
\text { y del paciente }[F=6.88, \\
p=.001], \text { reduciendo la } \\
\text { gravedad de los } \\
\text { síntomas de los } \\
\text { pacientes [ } F=4.65, \\
p=.01] \text { y el número de } \\
\text { reingresos }[F=4.78, \\
p=0] .\end{array}$ \\
\hline $\begin{array}{c}\text { Gräbel, } \\
\text { Trilling, } \\
\text { Donath, } \\
\text { Luttenberger } \\
(2010) \\
\text { VIII }\end{array}$ & $\begin{array}{c}\text { Estudio } \\
\text { transversal. } \\
404 \text { cuidadores } \\
\text { de pacientes con } \\
\text { demencia. } \\
\text { Alemania }\end{array}$ & $\begin{array}{c}\text { Determinar qué motiva a } \\
\text { los cuidadores en la } \\
\text { utilización de los GAM. } \\
\text { Determinar las } \\
\text { características de los } \\
\text { GAM. } \\
1\end{array}$ & $\begin{array}{l}\text { Los GAM que ofrecen } \\
\text { un intercambio de } \\
\text { experiencias y debates } \\
\text { abiertos cumplen con } \\
\text { los requisitos de los } \\
\text { cuidadores. } \\
\text { El grado de } \\
\text { "necesidad" }(p<.001) \text { y }\end{array}$ \\
\hline
\end{tabular}




\begin{tabular}{|c|c|c|c|}
\hline & & $\begin{array}{l}\text { Análisis de contenido y } \\
\text { análisis de regresión } \\
\text { logística binaria. }\end{array}$ & $\begin{array}{l}\text { la accesibilidad de los } \\
\text { GAM es un predictor } \\
\text { significativo para el uso } \\
(p=.005)\end{array}$ \\
\hline $\begin{array}{c}\text { Hemamalini, } \\
\text { Judie } \\
(2014) \\
\text { II }\end{array}$ & $\begin{array}{l}\text { Ensayo clínico, } \\
\text { con pre-test y } \\
\text { pos-test. } \\
240 \text { cuidadores } \\
\text { de personas que } \\
\text { sufrieron un } \\
\text { accidente } \\
\text { cerebro-vascular, } \\
120 \text { del grupo de } \\
\text { control y } 120 \text { del } \\
\text { grupo } \\
\text { experimental } \\
\text { aleatorizado. } \\
\text { India }\end{array}$ & $\begin{array}{l}\text { Evaluar el efecto de los } \\
\text { GAM en los puntos } \\
\text { fuertes del sistema } \\
\text { familiar entre los } \\
\text { proveedores de atención } \\
\text { a sobrevivientes de } \\
\text { accidentes } \\
\text { cerebrovasculares } \\
\text { (ACV). } \\
3 \\
\text { Análisis de la varianza (t- } \\
\text { test). }\end{array}$ & $\begin{array}{c}\text { Después de } 3 \text { meses, } \\
\text { hubo una diferencia } \\
\text { significativa ( } t=37.58 ; \\
p=.001 \text { ) en las } \\
\text { fortalezas del sistema } \\
\text { familiar entre el grupo } \\
\text { experimental [44.73 } \\
\text { (SD 5.83)] y el grupo } \\
\text { control [22.08 (SD } \\
3.07 \text { )]. } \\
\text { GAM intervención de } \\
\text { enfermería eficaz para } \\
\text { fortalecer el sistema } \\
\text { familiar de cuidadores } \\
\text { de supervivientes de } \\
\text { ACV. }\end{array}$ \\
\hline $\begin{array}{l}\text { Laakkonen, } \\
\text { et al. } \\
\text { (2013) } \\
\text { II }\end{array}$ & $\begin{array}{l}\text { Ensayo clínico } \\
\text { controlado } \\
\text { aleatorizado. } \\
136 \text { pacientes } \\
\text { con demencia y } \\
\text { sus cónyuges. } \\
\text { Finlandia }\end{array}$ & $\begin{array}{c}\text { Examinar la eficacia de } \\
\text { los GAM en la } \\
\text { aceptación del rol y el } \\
\text { bienestar psicológico de } \\
\text { los cuidadores. } \\
2 \\
\text { Análisis de diferencias } \\
\text { con la prueba U de } \\
\text { Mann-Whitney. }\end{array}$ & $\begin{array}{l}\text { La aceptación del rol } \\
\text { fue más alta en el } \\
\text { grupo de intervención } \\
\text { [98.9 (SD 12.7)] que en } \\
\text { el control [93.0 (SD } \\
15.3)](p=.024) . \text { El } 90 \% \\
\text { del grupo experimental } \\
\text { respondió estar muy } \\
\text { satisfecho. }\end{array}$ \\
\hline $\begin{array}{l}\text { McKechnie, } \\
\text { Barker, } \\
\text { Stott } \\
(2014) \\
\text { IV }\end{array}$ & $\begin{array}{l}\text { Estudio mixto: } \\
\text { cualitativo y } \\
\text { estudio cuasi- } \\
\text { experimental. } \\
61 \text { cuidadores de } \\
\text { personas con } \\
\text { demencia, que } \\
\text { participan en un } \\
\text { GAM online. } \\
\text { Reino Unido }\end{array}$ & $\begin{array}{l}\text { Examinar el impacto de } \\
\text { un GAM online para } \\
\text { cuidadores de personas } \\
\text { con demencia. } \\
\qquad 2,4 \\
\text { Análisis de la varianza (t- } \\
\text { test) y análisis temático } \\
\text { de las entrevistas. }\end{array}$ & $\begin{array}{c}\text { Hubo una mejora en la } \\
\text { relación con la persona } \\
\text { con demencia a las } 12 \\
\text { semanas [de } 48.61 \text { (SD } \\
9.20 \text { ) a 51.36 (SD } \\
9.87 \text { ); } \mathrm{p}=.003 \text { ]. No hubo } \\
\text { cambios en la } \\
\text { depresión ni ansiedad. } \\
\text { Los entrevistados } \\
\text { informaron de } \\
\text { beneficios positivos. }\end{array}$ \\
\hline $\begin{array}{l}\text { Rodriguez- } \\
\text { Sanchez, et } \\
\text { al. } \\
\text { (2013) }\end{array}$ & $\begin{array}{l}\text { Ensayo clínico } \\
\text { controlado } \\
\text { aleatorizado. } \\
125 \text { cuidadores } \\
\text { de personas con } \\
\text { demencia o } \\
\text { cualquier otra } \\
\text { discapacidad. } \\
\text { España }\end{array}$ & $\begin{array}{l}\text { Evaluar el efecto de los } \\
\text { GAM sobre los } \\
\text { pensamientos } \\
\text { disfuncionales sobre el } \\
\text { cuidado, la calidad de } \\
\text { vida y la carga de los } \\
\text { cuidadores. } \\
2 \\
\text { Regresión logística } \\
\text { binaria. }\end{array}$ & $\begin{array}{c}\text { El grupo de } \\
\text { intervención mostró } \\
\text { una mejoría en la salud } \\
\text { mental (IC del 95\%: } \\
-5,95 \text { a }-0,70 ; p=.01) \text { y } \\
\text { en los pensamientos } \\
\text { disfuncionales sobre el } \\
\text { cuidado (IC del } 95 \% \text { : } \\
-10.60 \text { a }-1.09 ; p=.01) \text {. } \\
\text { Los hombres asistieron } \\
\text { más que las mujeres } \\
(p<.001) \text {. }\end{array}$ \\
\hline
\end{tabular}




\begin{tabular}{|c|c|c|c|}
\hline $\begin{array}{c}\text { Secanilla, } \\
\text { Bonjoch, } \\
\text { Galindo, } \\
\text { Gros } \\
(2011)\end{array}$ & $\begin{array}{c}\text { Mixto: } \\
\text { Observacional } \\
\text { descriptivo } \\
\text { transversal y } \\
\text { cualitativo. } \\
30 \text { cuidadores de } \\
\text { personas con } \\
\text { enfermedad de } \\
\text { Alzheimer. } \\
\text { España }\end{array}$ & $\begin{array}{c}\text { Analizar el nivel de } \\
\text { satisfacción con respecto } \\
\text { a los GAM y la } \\
\text { sobrecarga de los } \\
\text { cuidadores de personas } \\
\text { con Alzheimer. } \\
2,4 \\
\text { Análisis descriptivo y } \\
\text { observación participante. }\end{array}$ & $\begin{array}{c}\text { Se demuestra que se } \\
\text { favorece la } \\
\text { permanencia de los } \\
\text { usuarios en el propio } \\
\text { domicilio y se mejora la } \\
\text { calidad de vida de } \\
\text { éstos y de sus } \\
\text { cuidadores, aunque } \\
\text { éstos muestran } \\
\text { sentimientos de } \\
\text { codependencia. }\end{array}$ \\
\hline $\begin{array}{l}\text { Theurer, } \\
\text { et al. } \\
(2014) \\
\text { IX }\end{array}$ & $\begin{array}{l}\text { Cualitativo. } \\
65 \text { cuidadoras de } \\
\text { personas con } \\
\text { demencia, u } \\
\text { otros trastornos, } \\
\text { como } \\
\text { cardiopatías, } \\
\text { enfermedad } \\
\text { mental o } \\
\text { trastornos } \\
\text { músculo- } \\
\text { esqueléticos. } \\
\text { Canadá }\end{array}$ & $\begin{array}{l}\text { Describir el desarrollo de } \\
\text { un nuevo GAM para } \\
\text { cuidadores familiares, y } \\
\text { evaluar el proceso, } \\
\text { estructura y contenido de } \\
\text { la intervención y la } \\
\text { replicación y } \\
\text { sostenibilidad. } \\
1,2 \\
\text { Análisis de métodos } \\
\text { mixtos: grupos focales, } \\
\text { observación sistemática, } \\
\text { entrevistas individuales. }\end{array}$ & $\begin{array}{c}\text { Beneficios positivos, } \\
\text { incluyendo una } \\
\text { disminución en la } \\
\text { soledad, el desarrollo } \\
\text { de amistades y el } \\
\text { aumento de } \\
\text { habilidades de } \\
\text { afrontamiento, la } \\
\text { comprensión y apoyo. } \\
\text { Los participantes } \\
\text { informaron que } \\
\text { disfrutaban de la } \\
\text { variedad de los } \\
\text { componentes del GAM } \\
\text { (música, citas, } \\
\text { interlocutor y } \\
\text { fotografías). }\end{array}$ \\
\hline $\begin{array}{c}\text { Wang, } \\
\text { Chien, } \\
\text { Lee } \\
(2012) \\
\text { III }\end{array}$ & $\begin{array}{l}\text { Ensayo clínico, } \\
\text { con pre y post- } \\
\text { test. } \\
78 \text { cuidadores de } \\
\text { un familiar con } \\
\text { demencia, } 39 \text { en } \\
\text { cada grupo. } \\
\text { China }\end{array}$ & $\begin{array}{l}\text { Evaluar la eficacia de un } \\
\text { GAM para cuidadores de } \\
\text { un familiar con demencia } \\
\text { en la disminución de los } \\
\text { niveles de estrés, en } \\
\text { comparación con el } \\
\text { servicio de apoyo a la } \\
\text { familia estándar. } \\
2 \\
\text { ANOVA. }\end{array}$ & $\begin{array}{l}\text { Los participantes de los } \\
\text { GAM tuvieron mejorías } \\
\text { significativamente } \\
\text { mayores en los niveles } \\
\text { de angustia [F=19,86, } \\
p=.001] \text { y calidad de } \\
\text { vida que los del grupo } \\
\text { control [F=22.19, } \\
p=.001] .\end{array}$ \\
\hline $\begin{array}{l}\text { Wei, } \\
\text { et al. } \\
(2012)\end{array}$ & $\begin{array}{l}\text { Ensayo clínico, } \\
\text { con pre y post- } \\
\text { test. } \\
72 \text { cuidadores de } \\
\text { personas con } \\
\text { discapacidad } \\
\text { intelectual. } \\
\text { China }\end{array}$ & $\begin{array}{l}\text { Examinar la eficacia de } \\
\text { los GAM para personas } \\
\text { que cuidan a familiares } \\
\text { con discapacidad } \\
\text { intelectual con el objetivo } \\
\text { de mejorar su salud } \\
\text { física-psicológica y } \\
\text { apoyo social. } \\
2 \\
\text { Diferencias intragrupos } \\
\text { pre-postest con test de } \\
\text { Wilcoxon (z). }\end{array}$ & $\begin{array}{c}\text { La salud física y } \\
\text { psicológica ( } z=-6.50, \\
\mathrm{p}<.0001) \text { y el apoyo } \\
\text { social ( } \mathrm{z}=4.70, \\
\mathrm{p}<.0001) \text { de los } \\
\text { participantes en el } \\
\text { grupo experimental } \\
\text { mejoraron } \\
\text { significativamente a las } \\
4 \text { semanas de la } \\
\text { intervención. }\end{array}$ \\
\hline
\end{tabular}




\begin{tabular}{|c|c|c|c|}
\hline $\begin{array}{l}\text { Parker Oliver, } \\
\text { et al. } \\
(2017) \\
\text { I }\end{array}$ & $\begin{array}{l}\text { Metaanálisis. } \\
7 \text { revisiones } \\
\text { sistemáticas con } \\
10 \text { estudios sobre } \\
\text { cuidadores que } \\
\text { participan en un } \\
\text { GAM online. } \\
\text { USA }\end{array}$ & $\begin{array}{c}\text { Analizar el efecto de las } \\
\text { intervenciones de apoyo } \\
\text { grupal basadas en } \\
\text { Internet sobre el apoyo } \\
\text { social, la autoeficacia y } \\
\text { la carga del cuidador. } \\
1,2 \\
\text { Análisis usando un } \\
\text { software de metaanálisis } \\
\text { integral. }\end{array}$ & $\begin{array}{c}\text { Los GAM online tienen } \\
\text { un efecto positivo sobre } \\
\text { el apoyo social (IC del } \\
95 \%: 0.118-0.811, \\
\text { p=.009) y la } \\
\text { autoeficacia (IC: } 0.22- \\
0.659, p<.001) \text {, pero no } \\
\text { hubo diferencias en la } \\
\text { carga y, el tamaño y la } \\
\text { calidad de los estudios } \\
\text { son moderados. }\end{array}$ \\
\hline
\end{tabular}

SD: Desviación estándar.

Nivel Evidencia $\rightarrow$ I-II: Adecuada. III-IV: Buena a regular. V-VII: Regular. VIII-IX: Pobre.

Categorías $\rightarrow$ 1: Características de los GAM. 2: GAM en cuidadores. 3: GAM en la salud familiar. 4:

GAM para la persona dependiente.

\section{DISCUSIÓN}

Se ha llevado a término el análisis de los 12 artículos seleccionados para determinar cuáles son las características de los grupos de ayuda mutua y el impacto de esos grupos en los cuidadores familiares, las personas dependientes y la salud familiar, además de la sociedad o medio en el que se desarrollan.

\section{Características de los grupos de ayuda mutua}

El GAM se basa en apoyo de los compañeros, el uso de dinámicas de grupo y el empoderamiento de que los participantes tomen agencia activa en su vida. Principalmente se intenta animar a los participantes a identificar sus fortalezas y a mejorar sus habilidades para resolver problemas. Además, los GAM que ofrecen un intercambio de experiencias, la discusión abierta, información y asesoramiento cumplen los requisitos de los cuidadores familiares. ${ }^{(22,26)}$

Conjuntamente los GAM, apoyan a las familias a ser física, mental y socialmente activas, y promueven conocimiento para entender y gestionar la enfermedad, que incluye dimensiones tales como ayudar a los pacientes y sus cuidadores para identificar los problemas, el desarrollo de sus propias habilidades y mejorar su autoeficacia para la resolución de problemas y dominar su vida cotidiana. Por otro lado, proporcionan una sensación de tener algo en común con otros cuidadores, la validación de experiencias y oportunidades, es decir, dar y recibir ayuda. ${ }^{(29)}$

Además, existe otra manera de GAM, cómo indica McKechnie $V$, et al (25) en su estudio, el apoyo del cuidador mediado por ordenador.

Este tipo de GAM, ofrece una gama de posibles ventajas en comparación con los grupos de apoyo tradicionales cara a cara, incluida la accesibilidad y la posibilidad de adaptar a las necesidades individuales. Se centra específicamente en el apoyo mutuo online para los cuidadores de personas con demencia, y se utiliza para pedir consejo, compartir información, participar en las discusiones y sentirse apoyado.

Los participantes de este grupo de autoayuda online refirieron una mejora en la calidad de la relación con la persona con demencia. No hubo ningún cambio en la 
depresión o la ansiedad de los usuarios durante el período de estudio, pero los entrevistados informaron de una serie de experiencias y beneficios positivos del uso del foro, en comparación con los tradicionales GAM.

En general, se observa una mejoría significativa, en la comodidad, la obtención de un posible anonimato, la disminución de ansiedad y vergüenza producida por el contacto directo con otras personas y el intercambio de ideas y sentimientos, mucho mayor que en los GAM tradicionales.

No obstante, dichos grupos online necesitan mucha más investigación y tiempo para la obtención de los mismos beneficios que los presenciales, como por ejemplo, poder ofrecer educación para la salud por profesionales sanitarios ${ }^{(11)}$.

En definitiva los GAM para cuidadores deben concentrarse principalmente en debate moderado de experiencias de cuidado. Deben integrar elementos psico-educativos, en particular la información sobre la enfermedad y el tratamiento disponible, para saber cómo hacer frente a la situación de los pacientes. Para aumentar la utilización de grupos de apoyo, todos los cuidadores familiares no sólo deben ser informados acerca de la existencia y la accesibilidad de tales ofertas, sino también sobre las ventajas para sí mismos ${ }^{(29)}$.

\section{Los cuidadores familiares}

Se ha podido observar en el estudio de Wei Y-S, et al ${ }^{(30)}$, que un GAM de cuidadores familiares dependientes es una intervención eficaz para la promoción del estado de salud física y psicológica de los cuidadores, así como su apoyo social, proporcionando aquella red de relaciones sociales que las personas cuidadoras han ido perdiendo a lo largo del tiempo.

Wang $L-Q$, et al ${ }^{29)}$, afirman que, los participantes de los grupos de apoyo mutuo, tuvieron mejorías significativas en los niveles de angustia y calidad de vida. Estos hallazgos apoyan la eficacia de los grupos de apoyo mutuo para ofrecer apoyo psicosocial a los familiares cuidadores. Por lo tanto, debe ser convertido en un componente habitual de los cuidadores de las personas con dependencia en los cuidados de enfermería. Además, los resultados, indican la necesidad de enfermeras (como agentes de cuidado) para evaluar la salud del cuidador más tarde en el proceso de cuidar y ser consciente de su relación con el soporte emocional. Los resultados también destacan la importancia de que los cuidadores establezcan un sistema de auto-cuidado adecuado que proporcione apoyo emocional y ayuda física.

Rodriguez-Sanchez E. et al (26) defienden que la implementación de una intervención psicológica mejora la salud mental de los cuidadores de familiares a cargo, por lo que, el hecho de que el malestar emocional no aumente y que, incluso, disminuya significativamente, puede ser considerado como un importante logro.

El estudio de Laakkonen M-L, et al ${ }^{(24)}$, se centró en la gestión de la enfermedad y se vio cómo la intervención ayuda a aceptar el rol del cuidador.

Finalmente, Candy B, et al (20), nos afirman que las intervenciones que apoyan directamente a la familia o amigos, pueden ayudarlos a afrontar su función de cuidador y mejorar su calidad de vida. 


\section{La salud familiar}

El artículo escrito por, Hemamalini M, et al (23), habla sobre ello. Este artículo, refiere que los GAM tienen un impacto profundo en el sistema familiar, especialmente en su estructura de roles y las funciones de la familia. Los principales objetivos de las intervenciones familiares son disminuir los efectos negativos inmediatos de estrés que sienten los miembros de la familia, así como movilizar sus capacidades de afrontamiento de manera adaptativa. Los cuidadores familiares informaron que la intervención satisfacía sus necesidades de apoyo, disminuyendo algunas de sus demandas de cuidado y aumentando su confianza y capacidad de hacer frente a la situación del familiar dependiente. Por lo que, el estudio concluye que los GAM son una intervención de enfermería eficaz, recomendado para los cuidadores de pacientes dependientes para fortalecer el sistema familiar.

En definitiva, como afirman Chien WT, et al (21), los participantes de un GAM indicaron una mejoría significativamente mayor en la familia y el funcionamiento del paciente y el apoyo social percibido por las familias. Por lo tanto, los GAM suponen una intervención comunitaria efectiva para los cuidadores de personas dependientes y sus familias.

\section{Las personas dependientes}

En diferentes estudios, nombrados anteriormente, se ha podido observar que la disminución del estrés y la ansiedad de los cuidadores, el intercambio de sentimientos, situaciones y posibles actuaciones frente al cuidado de las personas dependientes, sumándole la información frente a este cuidado que ofrecen los GAM a los cuidadores, tiene una repercusión directa en los pacientes que ellos cuidan, al poder tener más capacidad para la mejora del estado de salud de los pacientes dependientes. Además, se suma una disminución de recaídas y de los síntomas de los pacientes y una disminución a los servicios sanitarios, gracias a la autonomía que ganan los cuidadores por la asistencia a los GAM. $(20,21,30)$

Por otro lado, Theurer $\mathrm{K}$, et al (28) describen grupos que van dirigidos a cuidadores junto con las propias personas dependientes, que revelaron, después de evaluar las entrevistas y observaciones estructuradas, varios temas, como la autodeterminación, el dar y recibir ayuda a otros pacientes, las discusiones de sus fortalezas y creencias, y la expresión de los retos a los que se enfrentan y cómo lidiar mejor con ellos.

También, como nos describe Secanilla $E$, et al (27), la decisión de ingresar a las personas dependientes en un centro residencial, es tomada de forma reflexiva y más distanciada, sin tantas emociones negativas, gracias a la asistencia de los cuidadores a GAM. Además, el grupo ayuda a intercambiar informaciones útiles sobre temas derivados del cuidado del enfermo y del autocuidado, lo cual permite la mejora en los pacientes dependientes.

\section{Sociedad}

Un tercio de los artículos (cuatro de los doce) se desarrollan en una sociedad oriental, tres de ellos en China y uno en la India. Las diferencias culturales entre una sociedad oriental y otra occidental repercuten a la hora de extrapolar los resultados. Por ejemplo, el estudio de Chien WT, et al ${ }^{(21)}$ se desarrolla en China con cuidadores masculinos (2/3), en contra de nuestro medio (donde predomina el rol del cuidador en 
las mujeres). Sin embargo, en el de Wang $L-Q$, et al ${ }^{(29)}$, fue una enfermera de práctica avanzada la que diseñó el protocolo para guiar el proceso del GAM y la capacitación del facilitador y cuidador-moderador, basado en la evidencia de la literatura sobre el GAM familiar en los países occidentales.

\section{Limitaciones y fortalezas}

Lo expuesto anteriormente es una de las limitaciones del estudio, aunque uno de ellos se basa en un GAM occidental. Por otro lado, sólo se han incluido los artículos completos disponibles, lo que implica que se han podido dejar de considerar otros artículos importantes sobre el tema.

Tres de los doce artículos elegidos son estudios de tipo observacional descriptivo transversal y/o cualitativo, que aportan información importante y relevante, pero, a su vez, presentan menor nivel de evidencia que los estudios de tipo experimental o las revisiones sistemáticas. Aun así, en más de la mitad de ellos se emplea una metodología de alto nivel de evidencia. Además, esta revisión bibliográfica se ha realizado por pares.

\section{CONCLUSIONES}

Los GAM pueden ayudar a reducir el malestar físico y psicológico de los cuidadores, fortaleciendo así el sistema familiar. Además, la asistencia de los cuidadores a GAM proporciona beneficio para las personas dependientes, con una disminución de recaídas.

Las características de los GAM se fundamentan en el apoyo entre sus miembros. También, se han encontrado GAM online, que ofrecen otras ventajas, como la accesibilidad.

No obstante, existen pocos estudios recientes en nuestro medio, por lo que se deberían realizar más, y con alto nivel de evidencia, para poder extrapolar los resultados a nuestra población y, finalmente, impulsar la implementación de los GAM en nuestra sociedad.

\section{REFERENCIAS}

1. Ullgren $\mathrm{H}$, Tsitsi $\mathrm{T}$, Papastavrou $\mathrm{E}$, Charalambous A. How family caregivers of cancer patients manage symptoms at home: A systematic review. Int J Nurs Stud [Internet]. $\quad 2018 \quad$ Sep 1;85:68-79. Disponible en: https://www.sciencedirect.com/science/article/pii/S0020748918301160

2. Tatangelo G, McCabe M, Macleod A, You E. "I just don't focus on my needs." The unmet health needs of partner and offspring caregivers of people with dementia: A qualitative study. Int J Nurs Stud [Internet]. 2018 Jan 1;77:8-14. Disponible en: https://www.sciencedirect.com/science/article/pii/S0020748917302183

3. Sandstedt P, Littorin S, Cröde Widsell G, Johansson S, Gottberg K, Ytterberg C, et al. Caregiver experience, health-related quality of life and life satisfaction among informal caregivers to patients with amyotrophic lateral sclerosis: A cross-sectional study. J Clin Nurs [Internet]. 2018 Dec 1;27(23-24):4321-30. Disponible en: http://doi.wiley.com/10.1111/jocn.14593 
4. Deek H, Hamilton S, Brown N, Inglis SC, Digiacomo M, Newton PJ, et al. Familycentred approaches to healthcare interventions in chronic diseases in adults: a quantitative systematic review. J Adv Nurs. 2016 May 1;72(5):968-79. Disponible en: http://doi.wiley.com/10.1111/jan.12885

5. Confederación Española de Organizaciones en favor de las Personas con Discapacidad Intelectual (FEAPS). Libro blanco sobre la dependencia resumen. Andalucía: FEAPS; 2014.

6. Losada A, Nogales-González, C, López J, Márquez-González M, JiménezGonzález E. Ayuda psicológica a cuidadores en diferentes escenarios. Información psicológica. 2013;105:60-77.

7. Alves de Oliveira F, Dos Santos M.A. Terapia comunitária como recurso de abordagem do problema do abuso do álcool, na atenção primária. Rev. LatinoAm. Enfermagem 19. 2011:821-30. Disponible en: http://www.scielo.br/pdf/rlae/v19nspe/21.pdf

8. López J, Crespo M. Intervenciones con cuidadores de familiares mayores dependientes: una revisión Psicothema. Ediuno. Ediciones de la Universidad de Oviedo. 2007;19(1):72-80. Disponible en: http://dialnet.unirioja.es/servlet/articulo?codigo=2231545\&info=resumen\&idioma=S PA

9. Conde J. L. Demencia y familiares cuidadores. Deterioro, sobrecarga y apoyo social. En Alberca R., López-Pous S. (eds.) Enfermedad de Alzheimer y otras demencias. Madrid: Editorial Médica Panamericana; 2006.

10.Domínguez-Orosco ME. La creación de grupos de ayuda mutua para familiares de personas dependientes. Revista Electrónica de Psicología Social Poiésis. 2012;23. Disponible en: http://www.funlam.edu.co/revistas/index.php/poiesis/index

11. Parker Oliver D, Patil S, Benson JJ, Gage A, Washington K, Kruse RL, et al. The Effect of Internet Group Support for Caregivers on Social Support, Self-Efficacy, and Caregiver Burden: A Meta-Analysis. Telemed e-Health [Internet]. 2017;23(8):621-9. Disponible en: http://www.ncbi.nlm.nih.gov/pubmed/28328392

12. Urrutia G, Bonfill X. PRISMA declaration: Aproposal to improve the publication of systematic reviews and meta-analyses. Med Clin(Barc). 2010; 135(11):507-511.

13. Medicine. Pubmed. [Internet]. USA; 2015. [actualizado 10 mayo 2015]. Disponible en: http://www.ncbi.nlm.nih.gov/pubmed

14. Base de datos/navegador/multibuscador de Elsevier B. Scopus. [Internet]. USA; 2015. [actualizado 1 mayo 2015]. Disponible en: http://www.scopus.com/

15. BIREME/OPS/ Cochrane en la Biblioteca Virtual en Salud (BVS). La Biblioteca Cochrane Plus. [Internet]. Barcelona; 2015. [actualizado 05 mayo 2015; citado 15 mayo 2015]. Disponible en: http://www.bibliotecacochrane.com/

16. El Centro de Recursos de Información de la Educación (ERIC). [Internet]. USA; 2015. [actualizado 14 mayo 2015; citado 15 mayo 2015]. Disponible en: https://www.ebscohost.com/us-high-schools/eric

17. American Psychological Association. PsycINFO. [Internet]. Washington; 2015. Disponible en : http://www.apa.org/pubs/databases/psycinfo/index.aspx

18. Biblioteca Nacional de Ciencias de la Salud (BNCS)-Instituto de Salud Carlos III (ISCIII). [Internet]. Madrid; 2015. Disponible en: http://bvsalud.isciii.es/

19.RedCASPe [Internet]. Institute of Health Sciences de Oxford (Universidad de Oxford y NHS R\&D): CASP: 2011 [Actualizado 26 febrero 2012]. Disponible en: http://www.redcaspe.org/drupal/?q=node/8

20. Candy B, Jones L, Drake R, Leurent MK B. Intervenciones para el apoyo de los cuidadores no profesionales de pacientes en la fase terminal de su enfermedad. Cochrane Database of Systematic Reviews. [Internet]. 2011. Disponible en: 

$\underline{s}$

21. Chien WT, Chan SW. The effectiveness of mutual support group intervention for Chinese families of people with schizophrenia: A randomised controlled trial with 24-month follow-up. Int J Nurs Stud [Internet]. 2013 Oct;50(10):1326-40. Disponible en: http://www.ncbi.nlm.nih.gov/pubmed/23433723

22. Gräbel E, Trilling A, Donath C, Luttenberger K. Support groups for dementia caregivers--predictors for utilisation and expected quality from a family caregiver's point of view: a questionnaire survey part I*. BMC Health Serv Res [Internet]. 2010;10:219.

Disponible

en: http://www.pubmedcentral. nih.gov/articlerender.fcgi? artid=2922206\&tool=pmcentre z\&rendertype $=$ abstract

23. Hemamalini M, Judie A. Psycho social intervention - An effective strategy in improving the family system strengths among the caregivers of stroke survivors. Int $\mathrm{J}$ Pharm Clin Res International Journal of Pharmaceutical and Clinical Research. [Internet]. 2014; 6(4):336-40. Disponible http://www.scopus.com/inward/record.url?eid=2-s2.084907862121\&partnerID=tZOtx3y1

24. Laakkonen M-L, Savikko N, Hölttä E, Tilvis R, Strandberg T, Kautiainen H, et al. Self-management groups for people with dementia and their spousal caregivers. A randomized, controlled trial. Baseline findings and feasibility. Eur Geriatr Med. 2013;4: 389-393.

Disponible

en: https://www.ncbi.nlm.nih.gov/pmc/articles/PMC3492198/

25. McKechnie V, Barker C, Stott J. The effectiveness of an Internet support forum for caregivers of people with dementia: a pre-post cohort study. J Med Internet Res [Internet]. 2014;16(2):e68. Disponible en: http://www.pubmedcentral.nih.gov/articlerender.fcgi?artid=3961748\&tool=pmcentre z\&rendertype $=$ abstract

26. Rodriguez-Sanchez E, Patino-Alonso MC, Mora-Simón S, Gómez-Marcos MA, Pérez-Peñaranda A, Losada-Baltar $A$, et al. Effects of a psychological intervention in a primary health care center for caregivers of dependent relatives: a randomized trial. Gerontologist [Internet]. 2013 Jun; 53(3):397-406. Disponible en: http://www.scopus.com/inward/record.url?eid=2-s2.084877628089\&partnerID=tZOtx3y1

27. Secanilla E, Bonjoch M, Galindo M, Gros L. La atención al cuidador. Una visión interdisciplinaria. European Journal of Investigation in Health, Psychology and Education. [Internet]. 2011. Disponible en: https://formacionasunivep.com/ejihpe/index.php/journal/article/view/20/7

28. Theurer K, Wister A, Sixsmith A, Chaudhury H, Lovegreen L. The Development and Evaluation of Mutual Support Groups in Long-Term Care Homes. J Appl Gerontol. SAGE Publications Inc. [Internet]. 2014; 33(4):387-415. Disponible en: https://www.ncbi.nlm.nih.gov/pubmed/24781963

29. Wang L-Q, Chien W-T, Lee IYM. An experimental study on the effectiveness of a mutual support group for family caregivers of a relative with dementia in mainland China. Contemp Nurse [Internet]. 2012 Feb; 40(2):210-24. Disponible en: http://www.ncbi.nlm.nih.gov/pubmed/22554214

30. Wei Y-S, Chu H, Chen C-H, Hsueh Y-J, Chang Y-S, Chang L-I, et al. Support groups for caregivers of intellectually disabled family members: effects on physicalpsychological health and social support. J Clin Nurs [Internet]. 2012 Jun; 21(1112):1666-77. Disponible en: http://www.ncbi.nlm.nih.gov/pubmed/22340134 
